\title{
Challenges of Interoperability and Integration in Education Information Systems
}

\author{
Kire Jakimoski \\ FON University, Faculty of Informatics, Skopje, Republic of Macedonia \\ E-mail: kire.jakimoski@fon.edu.mk
}

\begin{abstract}
In this work detailed research and analysis of the challenges of integration and interoperability in education information systems is presented. The integration methods and techniques are examined, as well as interoperability frameworks and challenges in the last 15 years. The work is also driven by sharing of assessment data for the purpose of efficient personalization of learning environments.
\end{abstract}

Keywords: education information system, enterprise application integration, interoperability, service oriented architecture

\section{Introduction}

Interoperability is defined by [1] as the capability to communicate with peer systems and right to use the functionality of peer systems. So, when interoperability is established between the systems, it means that they are related together with removed incompatibilities between them. The concepts of interoperability and integration are in different abstract levels and with separate objectives. Although both of them are significant for collaboration and seamless information flow, they are established on separate principles. The goal of integration is to collect and obtain information from a number of systems for some supposed system that asked for this information [2]. The aim of the interoperability on the other hand is to focus on continuous and seamless communication and exchange of information between cooperative systems [1]. From a different point of view, interoperability could be defined as the capacity of an autonomous system to exchange considerable information and start actions that belong to other systems in order to operate together and accomplish a common advantage.

Hence, interoperability is a capability of various systems to share data or functionalities and it became very actual topic for educational technologists [4]. The growing attention for interoperability is driven by the need for making the content accessible in different systems as well as by collaborative and cross-organizational learning and teaching [5]. In educational sectors there are a lot of included people like students, employees, etc. As a result of that, interconnection between various departments in order to exchange information is more than needed nowadays (Biggs, 2011). So integration could enable exchanging and sharing information to different departments in the educational sector [6]. Per example, university system includes communication with the extended campuses, university management department, student information department and library department [7].

In the available researches there are already proposed solutions to overcome this kind of challenges. School Interoperability Framework or SIF is proposed by the SIF Association which is an industry initiative to enable interoperability and data sharing [8]. SIF Implementation Specification NA 3.3 is released since May, 2015. Education Management Information System Interoperability Framework or EMIF is also presented from the China's government. 
SIF (School Interoperability Framework) is open specification for data sharing assigned for academic institutions from kindergarten through Grade 12 (K-12) [9]. This specification is mainly used in the USA, UK, Canada, and Australia. But, recently it is progressively being implemented in India as well as other countries around the globe. SIF specification consists of two key parts, SOA (Service-Oriented Architecture) specification aimed for sharing information between institutions, and XML specification aimed for modeling educational data according to the educational locale. SIF defines a group of definitions and rules for application to share information crosswise K-12 schools [10]. In the past years a lot of schools systems have accepted SIF in order to resolve the information interoperability in education [11]. However SIF has similar challenges that are present for the SOA specification and its data model.

Educational Information Technology Standardization that is part of the Ministry of Education of China released Education Management Information System Interoperability Framework (EMIF) to work out the challenges of sharing data and integrating different colleges and departments [12].

Anyway, there are still a lot of challenges that needs to be solved. In both SIF and EMIF concepts of interoperability, the communications models are founded on complex systems connection mode. Interoperability is achieved for each application over special connection codes for its database. So, in order to have development of the education management information systems, methods of complex systems connection mode should not be used, as well as implementation model of independent solutions [13].

Existing proposals are also not highly efficient if there is transmission of large data portions [14]. This kind of solutions also closely couples communication and information semantics. It is not useful in practical applications, because if the communication model is modified, information model will experience also a lot of changes. That is why in this work design, development and evaluation of a technical framework is designed for the adoption of the concept of SOA (Service Oriented Architecture) in integrating various EMIS in tertiary education [15]. The framework will provide a platform for seamless integration of different EMIS in order to resolve interoperability problems.

\section{Interoperability and Integration}

Most interactions in the education are based on interoperability amongst a diversity of data management systems, content repositories, and software applications [16]. In this kind of interactions three common types of activities are included [17]:

- Integrated usage of the digital content needed for learning and assessment in an array of platforms;

- Exchange of administrative and academic data between the software applications and databases in order to evaluate performance and maintain administrative reporting;

- Integration of administrative and educational applications between themselves and also with the local and system-wide enterprise software systems.

In order to maintain this kind of activities, main parts of the educational systems (teaching staff, students, administration) must follow standards for expressing digital content, and student and school data; for programming interfaces for applications; and for communicating between applications [18]. 


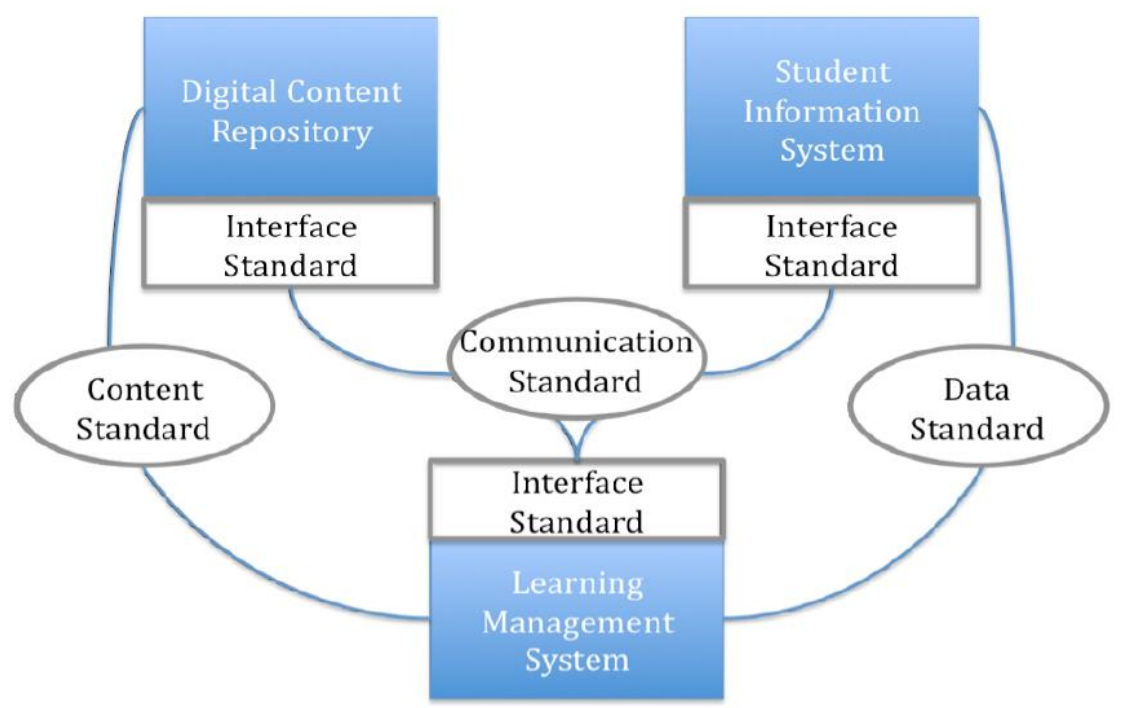

Figure 1. Educational System Infrastructure

Figure 1 is presenting the power of interoperability standards to smooth out the progress of the usage of content and data exchange across applications, and to decrease the effort of the developer that is needed to assemble data or content in the same time increasing the consumer preferences. According to the author in [19], the value of the applications is higher when more applications are using the standards. Educational system infrastructure in Figure 1 is using transport protocols to establish connections between systems and applications in order different components of the networked system to have transfer of content of data, and interface rules or APIs (Application Programming Interfaces) with instructions for using the general functions of an application [19].

This section is expected to clearly describe integration with focus on achieving interoperability or exchange of data across heterogeneous applications. You need to exhaust arguments in this section by describing the issues, prospects and predicaments (as it relates to integration for interoperability).

Integration of applications that are utilizing the content and data is supported by standard formats for data and content. Applications in education have to be integrated with identity management systems in order to enable authentication and authorization of the users when performing actions [20]. Educational standards for integrating applications involve PESC Data Transport Standard [21], IMS Learning Tools Interoperability [22], SIF Agent and SIF Zone Integration Server standards [23].

\section{Application Integration Methods}

The goal of application integration according to is to bring data or a function from one application together with that of other application that mutually offers almost real-time integration [24]. Per example, this can involve business-to-business integration, web integration, customer relationship management systems that could be integrated with a company's backend applications etc.

There are various methods for the application integration that are accepted by different organizations. Application integration could be realized with a reusable framework bringing together a group of technologies. There are three forms of enterprise application integration, vertical integration, horizontal integration, and mixed method integration [25]. Vertical integration is between systems that are on different levels of control and management in one organization. Horizontal integration is between different systems that 
support different functional areas, and mixed method integration is between systems of different organizations.

\subsection{Vertical Integration}

Vertical integration happens when systems that are implemented at various administrative levels of some organization are integrated. Although it is not unusual to integrate the functionality at various levels of an operational system into one single application, it is normal to have various systems implemented to refer to business functions at various management levels [25].

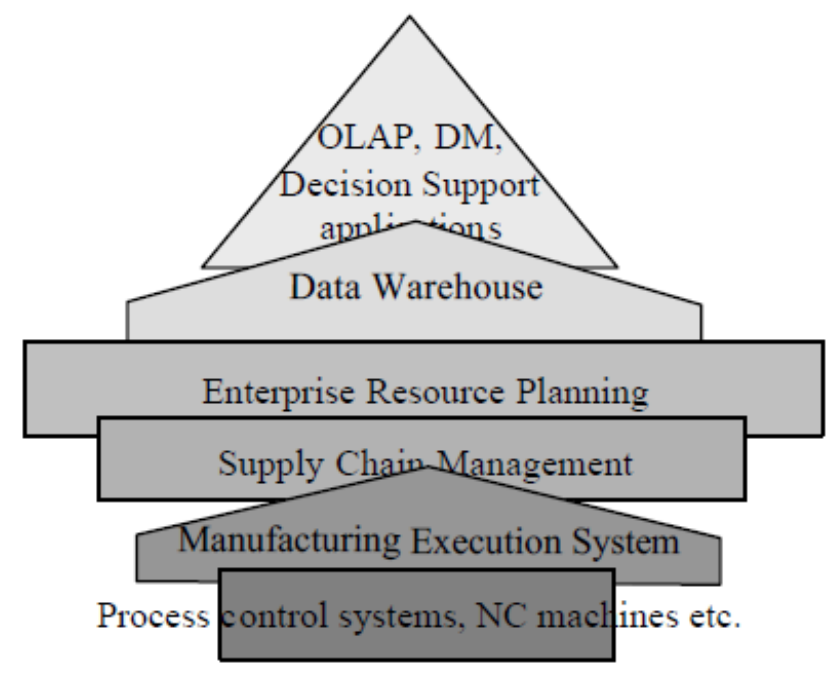

Figure 2. Vertical Integration of Different Layers

Therefore, vertical integration might require data flow in both directions between candidate applications. In order to analyze, improve and complement the data in the process of vertical integration a number of steps that include user interaction might be needed. Vertical integration could be achieved using extensive variety of technologies and approaches as well as products based on them [25]. But, despite what product or mixture of programming and products is utilized, the integration solution is affected by the economy and the stage of the assumed sophistication of the solution.

If vertical interoperability is analyzed in the context of the School Interoperability Framework (SIF), SIF agents at various levels of an organization communicate using a SIF zone. So, in this case vertical interoperability includes data gathering from multiple agents - upward, or publishing of data to multiple agents - downward. Per example, a state entity might need to publish teacher certification data to districts. Three parts of the SIF specification that deal directly with vertical interoperability are:

- Student Locator object;

- Vertical Reporting object;

- Data Warehouse object.

\subsection{Vertical Integration}

With horizontal integration organizations attempt entire set of activities to be optimized (purchasing, order entry, shipment, production etc.) in that way minimizing the production costs and lead-time, and maximizing the value for the customer at the same time. Figure 3 presents four processes and each of them spans several processes or 
applications. The gray area of the figure is describing the organization that is analyzed. Rectangles, on the other side, present the sub-processes or applications [25]. Process that is numbered with one is taking place totally in the organization, but the process with number two is starting from inside the organization and it is finishing outside of it. Process with the number three is starting from outside and it is finishing inside the organization. Then, process with number four is leaving the organization at one position, but comes back prior to its finish. Example for number four could be a case when individual company is responsible for delivery before the original company verifies that the invoice have been salaried. Key challenge of the horizontal integration is to connect various systems that contain the groups of legacy systems and recently developed applications into entire processes.

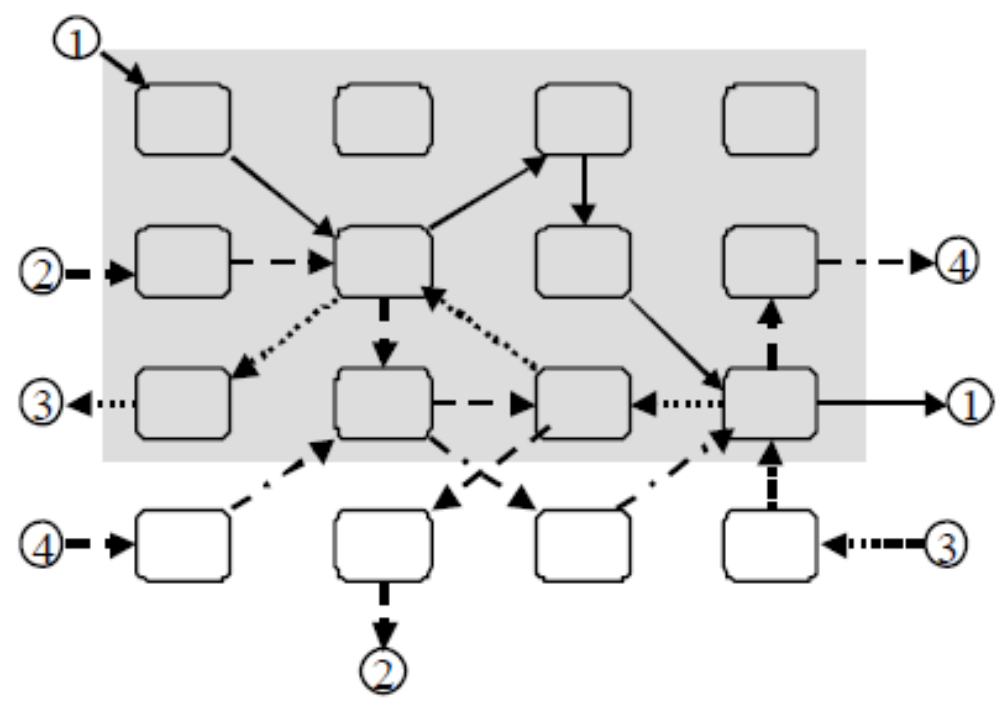

Figure 3. Example of Horizontal Integration

Horizontal integration could be achieved at a reasonable level using the EAI (Enterprise Application Integration) products maintaining the application integration level and levels above it as referenced. Although a lot of middleware products facilitate integration of applications utilizing known ERP (Enterprise Resource Planning) packages and legacy applications with familiar interfaces, an entire plug-and-play situation is still not possible.

\subsection{Mixed Method Integration}

The main challenge in mixed method integration is to get the data operations of one organization's application matching with another organization's application. Different assortment of priorities and requirements is involved as a result of the changed focus of application integration from within one company to multi-enterprise integration. Some of the requirements are modifications of approaches that have progressed for EAI, although there are still a lot of differences. One of them is the higher stress that is placed on security matters. Other type of difference is the increased need for an integrated support for Service Oriented Architectures (SOA). Dissimilarity between EAI solutions and application servers becomes more unclear in the mixed method integration, and as a result of this a new IAI (Internet Application Integration) is developed [25]. 


\section{Application Integration Techniques}

\subsection{Enterprise Application Integration}

The importance of integration of applications increased a lot since it became highly essential to let the automated systems to interact. So, the existing systems that provide interoperability have to be integrated and they would be more like Figure 4 .

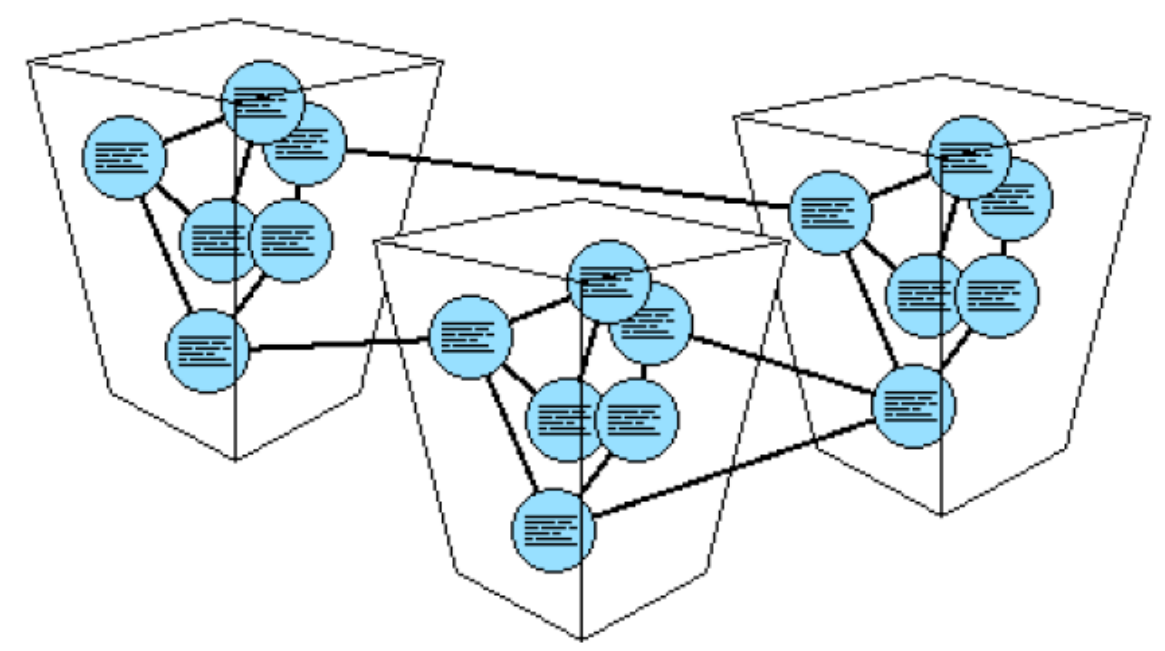

Figure 4. Integrated company

Enterprise Application Integration (EAI) covers each part of an enterprise system and it involves architecture, software, hardware and processes (Enterprise Architecture 2015). EAI requirements include [26]:

$\circ$ Business Process Integration (BPI). It includes process modeling, process management, and workflow.

- Application Integration. It involves CRM (customer relationship management) systems, business-to-business integration, web integration, and interactive web sites with multiple business systems.

- Data Integration. The data that is included must also be integrated in order the above mentioned integrations to work properly.

- Platform integration. This integration is dealing with tools and processes that are necessary to permit the systems to communicate.

All above mentioned integrations are very important for complete implementation of Enterprise Application Integration.

Two kinds of integration architecture are present in EAI, direct PTP (point-to-point) and middleware-based.

Point-to-point EAI integration architecture is traditional and basic approach. In theory, usually the number of integration points is doubled comparing it to the number of applications. This is presented in Figure 5, where 5 applications require 10 points of integration. 


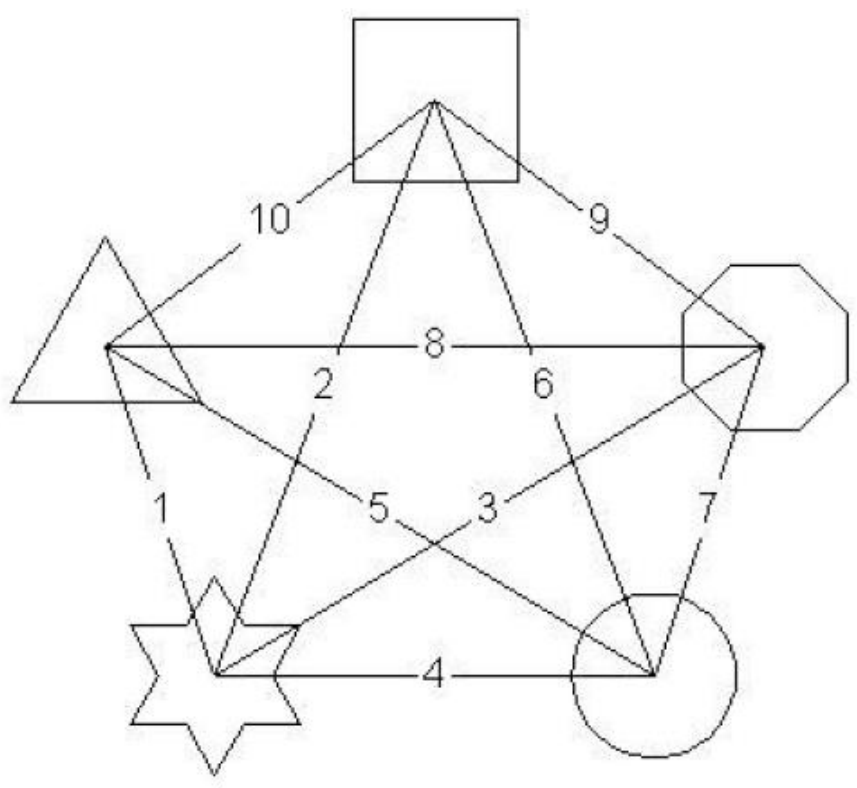

Figure 5. Point-To-Point EAI Integration Architecture

This could be avoided with an available intermediate layer that could separate the modifications between applications, efficiently decreasing the coupling. It can be done with using middleware [26].

Hence, middleware is used for mediation between applications, providing generic interfaces. In this way, applications can transmit messages to each other. Logical representation of the middleware integration can be seen in Figure 6. [27]. In this case five applications have now only five integration points, in that way halving the cost. Applications could be added or replaced in a way that doesn't influence on others. Operations like transforming, routing, aggregating, converting, and separating on the passed data could be performed by the middleware. Anyway, there is added difficulty during the setup up process of the middleware, as well as during the conversion of the applications to utilize the middleware APIs.

Application integration is supported by many commercial middleware products that are accessible in the market. Tools that are business process oriented and more advanced are typically referred to as EAI (Enterprise Application Integration) software [28].

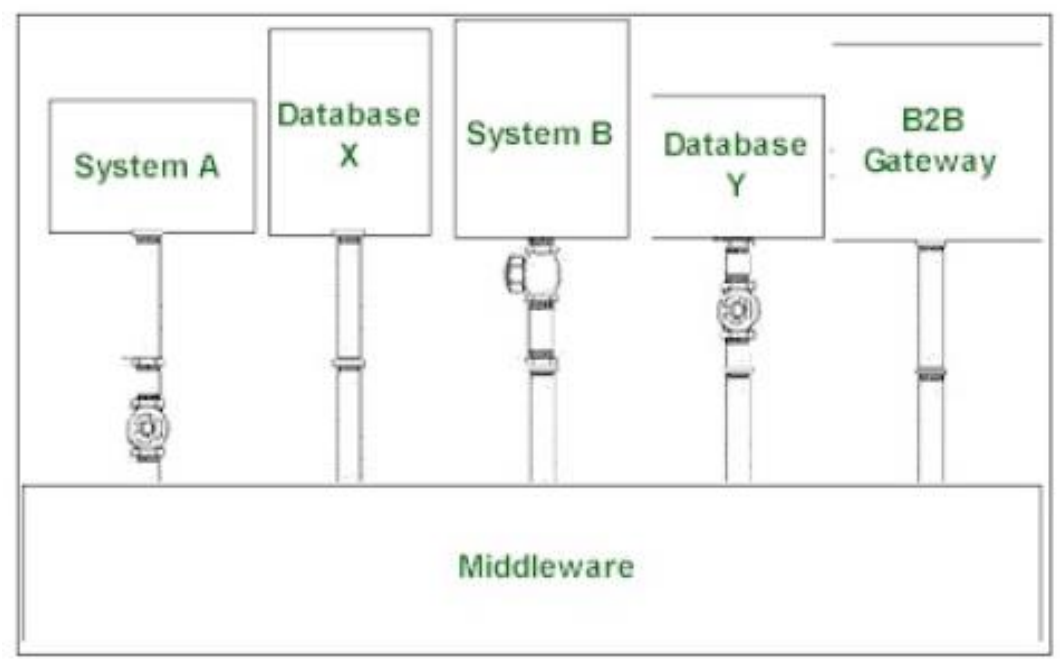

Figure 6. Integration Using Middleware 
EAI products entirely or partly automate different parts of the process that facilitates custom built and packaged business applications to exchange business level information. However, Enterprise Application Integration (EAI), which can be described as middleware of technologies and services, is argued by the authors in [29] as an approach to system or application integration. Middleware, providing enhanced support for EAI [30], is described as a technology stack that provides a platform for information exchange between applications in a distributed application environment [31]. Thus, EAI is found relevant as a technology to integrate different EMIS. This observation is strengthened by the argument of the authors in [32] that EAI allows multiple information systems within higher education institutions to be integrated to facilitate cross-platform information exchange and re-use. However, the fact that EAI provides core support for business process integration more than information integration [33] makes it inadequate for this research.

\subsection{Service Oriented Architecture}

There is no generally acceptable definition for SOA [34] as different researchers have looked at SOA from three different perspectives. From a business perspective, SOA is seen as a set of services; from an architectural perspective, SOA is a set of architectural principles, pattern and criteria; and from an implementation perspective, SOA is a complete programming model with standard, tools and technologies. In SOA, the service model has three components: Service Provider, Service Registry, and Service Requester; three operations in SOA: publish; find; and bind; and three XML based standard protocols: WSDL, UDDI and SOAP [13]. The interaction between the service components, operations and protocols can be viewed with a scenario where the Service Provider publishes services to the Service Registry using the WSDL. Then, the Service Requester finds the published service by performing service discovery operation on the service registry using the UDDI protocol. Once the service is discovered, the Service Requester then bind and invoke the appropriate service from the Service Provider using the SOAP over HTTP.

Anyway, there are other styles of software architectures that are alternative to the use of SOAP, which include the Extensible Mark-up Language-Remote Procedure Call (XML-RPC) and Representational State Transfer (REST) among others [35]. While SOAP is a traditional standard based web service technology, conceptually complex with a steeper learning curve and relatively "heavy-weight", REST lacks standard support for security and unusable in a distributed computing environment [36]. Meanwhile, the XMLRPC requests are stateless, synchronous and its use of HTTP protocol to transport XML data makes it less secured and relatively heavier than the XML [35].

Hence, it can be conclude here that in education systems, where education institutions use different EMIS as enterprise information system to manage academic activities at different levels, SOA can be adopted to reduce the complexity of integrating systems. Though, authors in [34] argued for a model of SOA adoption following his argument that there is limited academic research work on SOA adoption and issues of SOA implementation, there is the need to examine the adoption of SOA in EMIS within the context of architectural and business or organization perspectives.

\section{Interoperability Frameworks}

There are several different types of interoperability frameworks, like European Interoperability Framework (EIF), School Interoperability Framework (SIF), eGovernment Interoperability Framework, Enterprise Interoperability Framework, Standards and Interoperability (S\&I) Framework, Health Level 7 interoperability framework. 


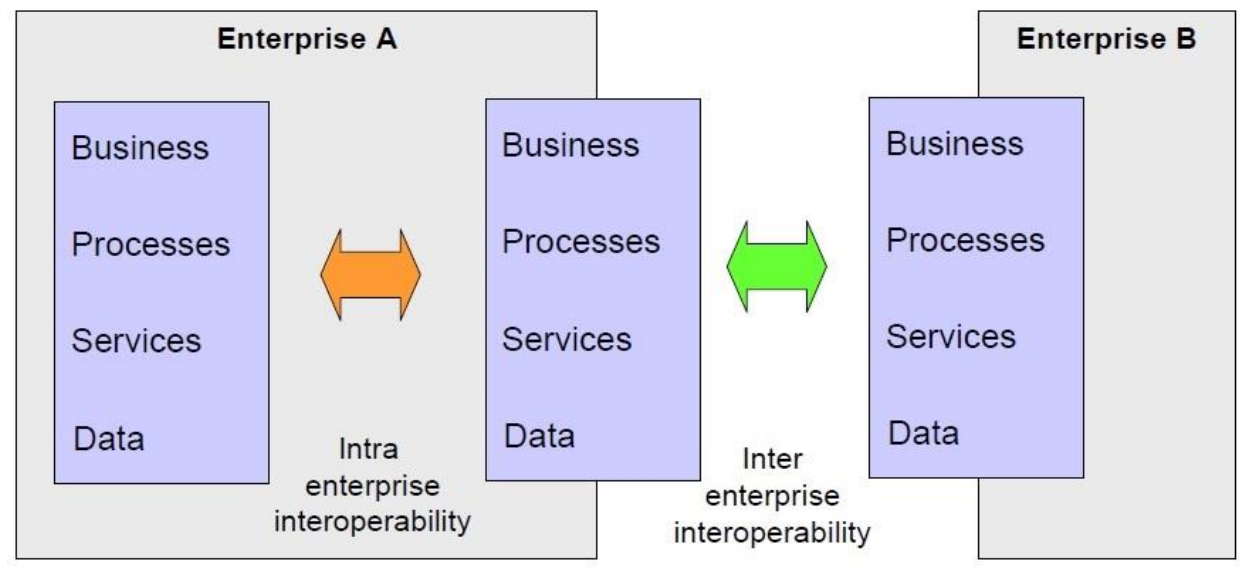

Figure 7. Interoperability Concerns

European Interoperability Framework presents a set of recommendations that identify how Administrations, Business and Citizens communicate with each other inside the EU and across the member states borders. The aim of e-Government Interoperability Framework is to enable the seamless flow of information across government or public service organizations.

Enterprise Interoperability Framework is utilized as a guideline for gathering and structuring knowledge or solution for enterprise interoperability. It has three basic dimensions, interoperability concerns, interoperability barriers and interoperability approaches. Interoperability concerns are presented in Figure 7 and they defined the content of interoperation that might occur at different levels of some enterprise. Generally, four interoperability concerns are recognized in the domain of Enterprise Interoperability, Data, Service, Process and Business [37]. Interoperability barrier is a basic concept when defining the interoperability domain, and three categories of barriers are recognized: conceptual, technological and organizational [38]. Interoperability approaches characterize the different ways in which barriers could be removed (unified, integrated and federated).

S\&I Framework includes associated community of participants from the private and public sectors that enables functional exchange of health information through providing tools, services and guidance. Health Level-7 or HL7 is a set of international standards for transmission of clinical and administrative data between software applications that are utilized by different healthcare providers.

One of the most used interoperability frameworks is School Interoperability Framework or SIF. It is an open specification for data sharing of academic institutions from kindergarten to workforce. This framework is mostly used in the USA, Canada, United Kingdom, and Australia, but it is also developing in India and other countries as well. Two main elements are included in this framework. The first one is an XML specification for modeling educational data that is identifiable to the educational environment (like Australia, United Kingdom or North America). The other main element is a SOA (Service-Oriented Architecture) specification for sharing the data between institutions. School Interoperability Framework was designed when SOAP, web services and namespaces standards were not developed like nowadays. Therefore, the 3.0 Infrastructure specifications completed the transformation to a SOA specification utilizing up-to-date tools. The release of the SIF Implementation Specification 3.0 was announced by the SIF Association in August 2013. This specification fully supports RESTful Web Services and SOAP-based protocols. Transport of different data models is allowed with the 3.0 infrastructure including from the ones from the other global SIF communities. 


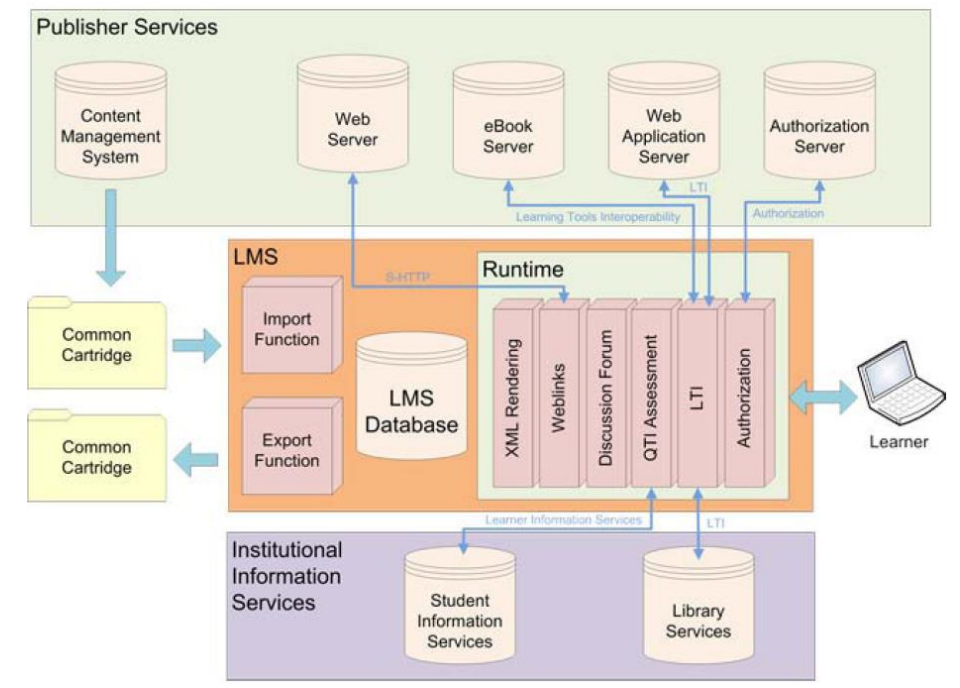

\section{Figure 8. Integration of Software Applications and Content Sources}

Web service architecture is defined with the PESC Data Transport Standard and it facilitates applications to respond and send requests utilizing standard web service protocols. SIF Agent or SIF Web service is specified by the SIF standard in order to share data inside a SIF Zone. SIF Zone is a logical collection of applications which Agents could communicate between each other through a SIF Zone Integration Server.

Individual learning applications could be integrated using the IMS Learning Tools Interoperability standard with platforms like LMSs, library information systems, eReaders, or other web applications. IMS Learning Tools Interoperability standards also deal with interoperability among web-based, externally hosted applications. The function of these standards in the educational computing environment is presented in Figure 8 [19]. Three main parts of the system in Figure 8 are LMS, Institutional Information Services and Publisher Services which are integrated together.

\section{Interoperability Challenges in Education Systems}

The success of an education system is dependent on the effectiveness of its Information System's ability to provide support for sharing, storing, classifying and use of information. It therefore means that several EMIS have to be integrated to address the holistic challenge of effectively managing the education system. For instance, a faculty member in a tertiary institution checking the profile of a student in the Student Information System (SIS) may as well want to overview the courses that the student has registered for during the session through the Course Registration System (CRS). This is feasible when the CRS is integrated with the SIS and are made to interoperate by exchanging data.

However, authors in [13] have argued that each of these EMIS that are used in the education system are developed with different operating systems, programming languages and technical standards, which make them to have different information and communication semantics. For instance, the SIS is developed with the J2EE and the CRS is developed with the .NET platform; these are applications with different platform and languages and are required to interoperate and exchange data. The resulting effect makes it difficult to integrate EMIS of different platforms and as such, it is almost impossible for EMIS to achieve the stated objectives of effective information management within education system.

It is only appropriate; therefore, to examine the arguments of the authors in [39] on the need to take a closer look at the structure and characteristics of data that are used by 
EMIS is a bid to plan an appropriate means and technologies for integrating different EMIS together.

Offering interoperability in education introduces also challenges with authentication of the users and authorization of their activities, although this kind of technical and policy matters are not exclusive to education. Legal and regulatory subjects that are present when interoperable data, programs, and content in education is managed have their analogues across IT industry sectors.

Digital rights management is another interoperability challenge for EMIS. Management of the digital rights is crucial for protection of intellectual property as well as for preserving the readiness of the software developer or the content creator to present their outcome in open educational systems. Existing interoperability standards offer a format for expressing license constraints that schools and librarians have to manage in order to share out software or content and allow students and instructors to utilize them. Software that utilizes these resources for expressing rights and permitting enforcement has to implement procedures that permit or forbid the usage of content as appropriate.

\section{Conclusion}

Education information system interoperability models are implementing the interoperability of educational information systems. The most important research results in this area are analyzed in this paper. Enterprise Application Integration (EAI) techniques and methods are also reviewed as very important tools for any company in the IT field. It can be concluded here that EAI is found relevant as a technology to integrate different EMIS.

Furthermore, key interoperability frameworks that are popular are reviewed in this work with more details for one of the most used interoperability framework School Interoperability Framework or SIF. Finally, the opened research challenges in the interoperability process are researched in details.

\section{References}

[1] D. Chen, G. Doumeingts and F. Vernadat, "Architectures for enterprise integration and interoperability: Past, present and future", Computers in industry, vol. 59, no. 7, (2008), pp. 647-659.

[2] L. Cardoso, F. Marins, C. Quintas, F. Portela, M. Santos, A. Abelha and J. Machado, "Interoperability in Healthcare", Cloud Computing Applications for Quality Health Care Delivery, (2014), pp. 78-101.

[3] C. Agostinho, Y. Ducq, G. Zacharewicz, J. Sarraipa, F. Lampathaki, R. Poler and R. J. Goncalves, "Towards a sustainable interoperability in networked enterprise information systems: Trends of knowledge and model-driven technology", Computers in Industry, (2015).

[4] A. W. P. Fok and H. H. S. Ip, "Educational ontologies construction for personalized learning on the web", In Evolution of teaching and learning paradigms in intelligent environment, Springer Berlin Heidelberg, (2007), pp. 47-82.

[5] K. Tammets, K. Pata and M. Laanpere, "Implementing a technology-supported model for crossorganizational learning and knowledge building for teachers", European Journal of Teacher Education, vol. 35, no. 1, (2012), pp. 57-75.

[6] M. Yarime, G. Trencher, T. Mino, R. W. Scholz, L. Olsson, B. Ness and J. Rotmans, "Establishing sustainability science in higher education institutions: towards an integration of academic development", institutionalization, and stakeholder collaborations. Sustainability Science, vol. 7, no. 1, (2012), pp. 101113.

[7] V. E. Cabukovski, "Integrated Agent-Based University Information System", in ELML'10, Second International Conference on Mobile, Hybrid, and On-Line Learning, (2010), pp. 36-40.

[8] SIF Association, 2015. Available from: http://specification.sifinfo.org/, (2015).

[9] O. A. Ise, "Towards a Unified University Information System: Bridging the Gap of Data Interoperability", American Journal of Software Engineering, vol. 2, no. 2, (2014), pp. 26-32.

[10] School Interoperability Framework, Available at: https://www.a4l.org/Pages/default.aspx. (Accessed 02 October 2015), (2014).

[11] National Schools Interoperability Program,. "Interoperability in education, Australia", 2014. Available at: http://www.nsip.edu.au/systems-interoperability-framework. (Accessed: 2 October 2015), (2014). 
[12] W. Yanfeng, B. Xinlong and H. Zhengbing, "The structure of EMIF and its model for data exchange and message processing", In Education Technology and Computer Science, 2009. ETCS'09. First International Workshop on, IEEE, , vol. 3, (2009), pp. 170-173.

[13] Z. Dongdai, "SOA-based Education Information System Interoperability Model", Journal of Information \& Computational Science, vol. 7, no. 5, (2010), pp. 115-1174.

[14] K. Saleem and Z. Bellahsene, "New Challenges in Data Integration: Large Scale Automatic Schema Matching", (2007).

[15] N. Lopez, R. Casallas and J. Villalobos, "Challenges in creating environments for SOA learning", In Systems Development in SOA Environments, 2007. SDSOA'07: ICSE Workshops 2007. International Workshop on, IEEE, (2007), pp. 9-9.

[16] D. Dagger, A. O'Connor, S. Lawless, E. Walsh and V. P. Wade, "Service-oriented e-learning platforms: From monolithic systems to flexible services", Internet Computing, IEEE, vol. 11, no. 3, (2007), pp. 2835.

[17] P. McGee, "Learning objects across the educational landscape: Designing for knowledge sharing and generation", Educational Technology-Saddle Brook Then Englewood Cliffs NJ, vol. 46, no. 1, (2006), pp. 26.

[18] J. Roschelle, J. Kaput, W. Stroup and T. M. Kahn, "Scalable integration of educational software: Exploring the promise of component architectures", Journal of Interactive media in education, no. 2, (2010), pp. 6.

[19] E. Walker, "Primer on K-20 Education Interoperability Standards", Publication of the Software Information Industry Association, (2012).

[20] M. Lim, “U.S. Patent No. 6,728,884. Washington”, DC: U.S. Patent and Trademark Office, (2004).

[21] J. Farmer, "Report from a Meeting of Chief Technologists: Harmonizing Web Services Data Transport Standards for Teaching", Learning, and Support of Students, (2010).

[22] C. Severance, T. Hanss and J. Hardin, "Ims learning tools interoperability: Enabling a mash-up approach to teaching and learning tools", Technology, Instruction, Cognition and Learning, vol. 7, no. 3-4, (2010), pp. $245-262$.

[23] J. P. Leal and R. Queirós, "eLearning Frameworks: a survey", In Proceedings of International Technology, Education and Development Conference, (2010).

[24] D. S. Linthicum, "Enterprise application integration", Addison-Wesley Professional, (2000).

[25] B. Wangler and S. J. Paheerathan, "Horizontal and vertical integration of organizational IT systems", Information Systems Engineering, (2000).

[26] J. Fenner, "Enterprise Application Integration Techniques", (2003).

[27] Gartner, Available from: < http://www.gartner.com/technology/home.jsp>. [30 September 2015], (2015).

[28] T. Lidfeldt, "Välkommen till middleware och EAI", (1999).

[29] A. Fazlollahi, U. Franke and J. Ullberg, "Benefits of Enterprise Integration: Review, Classification, and Suggestions for Future Research", In Enterprise Interoperability, Springer Berlin Heidelberg, (2012), pp. 34-45.

[30] E. Bertino, L. D. Martino, F. Paci and A. C. Squicciarini, "Web Service Technologies, Principles, Architectures, and Standards", In Security for Web Services and Service-Oriented Architectures, Springer Berlin Heidelberg, (2010), pp. 9-23.

[31] C. Emmersberger and F. Springer, "Tutorial: Open source enterprise application integration-introducing the event processing capabilities of apache camel", In Proceedings of the 7th ACM international conference on Distributed event-based systems, ACM, (2013), pp. 259-268.

[32] N. Aserey and S. N. Alshawi, "A conceptual model of enterprise application integration in higher education institutions", (2013).

[33] L. D. Xu, "Enterprise systems: state-of-the-art and future trends', Industrial Informatics", IEEE Transactions on, vol. 7, no. 4, (2011), pp. 630-640.

[34] E. MacLennan and J. P. V. Belle, "Factors affecting the organizational adoption of service-oriented architecture (SOA)", Information Systems and e-Business Management, vol. 12, no. 1, (2014), pp. 71100.

[35] A. Ploscar, "XML-RPC vs. SOAP vs. REST Web Services in Java-Uniform Using Wswrappe", International Journal Comput, vol. 4, (2012), pp. 215-223.

[36] P. A. Castillo, J. L. Bernier, M. G. Arenas, J. J. Merelo and P. G. Sánchez, "SOAP vs. REST: Comparing a master-slave GA implementation", arXiv preprint arXiv: 1105.4978, (2011).

[37] C. Guglielmina and A. Berre, "Project A4 (Slide presentation)", ATHENA Intermediate Audit, Athens, Greece, (2005).

[38] M. Kasunic and W. Anderson, "Measuring systems interoperability: challenges and opportunities", Software engineering measurements and analysis initiative, (2004).

[39] H. Hua and J. Herstein, "Education management information system (EMIS): Integrated data and information systems and their implications in educational management", In annual conference of comparative and International Education Society, (2003). 


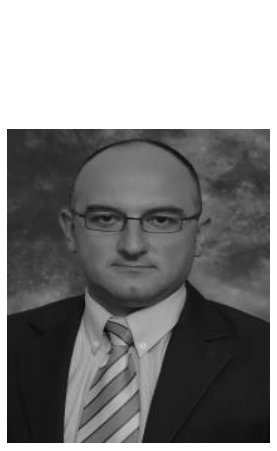

\section{Author}

Kire Jakimoski, was born in Gostivar, Republic of Macedonia, in 1979. He received his B.Sc. degree in the field of Telecommunications from the Military Academy "Mihailo Apostolski" in Skopje, R. Macedonia in 2002, M.Sc. degree in Electrical Engineering in the field of Telecommunications from the Ss. Cyril and Methodius University in Skopje, R. Macedonia in 2007, and $\mathrm{Ph} . \mathrm{D}$. in technical sciences from the Ss. Cyril and Methodius University in Skopje, R. Macedonia in 2013. From 2002 to 2006 he works as an Officer for Telecommunications in the Ministry of Defense in the Republic of Macedonia. From January, 2006 to March, 2012 he works as an adviser for information security in the Directorate for Security of Classified Information in the Republic of Macedonia. From March, 2012 he is with Faculty of Informatics, FON University in Skopje. Also, he is an author/co-author of around 30 published research papers and one book. He is an Assistant Professor at the Faculty of Informatics, FON University in Skopje, Macedonia. His research interests include Information and Communication Technologies, Wireless and Mobile Networks, Heterogeneous Wireless Networks, Information Security, Computer Networks, Security of Computer Systems. 
International Journal of Database and Theory and Application Vol.9, No.2 (2016) 